\title{
Scaling of tropical-cyclone dissipation
}

\author{
Álvaro Corral ${ }^{1 \star}$, Albert Ossó $^{2}$ and Josep Enric Llebot ${ }^{2}$
}

The influence of climate variability and global warming on the occurrence of tropical cyclones is a controversial issue $e^{1-10}$. Existing historical databases on the subject are not fully reliable ${ }^{9,11-13}$, but a more fundamental hindrance is the lack of basic understanding regarding the intrinsic nature of tropicalcyclone genesis and evolution ${ }^{14}$. It is known that tropical cyclones involve more than a passive response to changing external forcing ${ }^{15}$, but it is not clear which dynamic behaviour best describes them. Here we present an approach based on the application of the power dissipation index, which constitutes an estimation of released energy ${ }^{3}$, to individual tropical cyclones. A robust law emerges for the statistics of power dissipation index, valid in four different ocean basins and over long time periods. In addition to suggesting a description of the physics of tropical cyclones in terms of critical phenomena ${ }^{16,17}$, the scaling law enables us to quantify their response to changing climatic conditions, with an increase in the largest power dissipation index values with sea surface temperature or the presence of EI Niño phenomena, depending on the basin under consideration. In this way, we demonstrate that the recent upswing in North Atlantic hurricane activity does not involve tropical cyclones that are quantitatively different from those in other sustained high-activity periods before 1970.

One important characterization of a complex phenomenon is given by the fluctuations in the 'size' of the phenomenon over successive occurrences ${ }^{16-18}$. We refer to neither spatial size (area, volume) nor something like the Saffir-Simpson category ${ }^{19}$; rather, we seek a physically relevant measure of released energy. For a tropical cyclone (TC), a reasonable proxy for this energy has been proposed in ref. 3, using the power dissipation index (PDI), defined as

$$
\mathrm{PDI} \equiv \sum_{t} v_{t}^{3} \Delta t
$$

where $t$ denotes time and runs over the entire lifetime of the storm and $v_{t}$ is the maximum sustained surface wind velocity at time $t$. In available best-track records, measurements are provided at intervals of $\Delta t=6 \mathrm{~h}$. Note that in this paper the PDI value is associated with an individual TC, not with the total annual activity in some ocean basin ${ }^{3}$.

We analyse TC best-track records for several ocean basins: the North Atlantic and Northeastern Pacific (from the National Hurricane Center $^{20}$ ), and the Northwestern Pacific and the Southern Hemisphere (from the Joint Typhoon Warning Center ${ }^{21}$ ). We exclude the North Indian Ocean, owing to the small number of storms in the most reliable portion of its records. (For more details, see Supplementary Fig. S2.)

We show in Fig. 1a the PDI probability density, $D(P D I)$, normalized in the usual way $\left(\int_{0}^{\infty} D(\mathrm{PDI}) \mathrm{dPDI}=1\right)$, for all four basins. The distributions include all storms occurring during an extended period, either 1966-2007 or 1986-2007 as indicated in the legend. All four distributions (given vertical offsets for clarity) can be characterized by a power-law decay in their central regions,

$$
D(\mathrm{PDI}) \propto 1 / \mathrm{PDI}^{\alpha}
$$

where the exponent $\alpha$ is in between 0.95 and 1.25 (Supplementary Information, including Table S1). Deviations from the power law at small PDI values can be attributed to the deliberate incompleteness of the records for 'not significant' TCs, whereas the more rapid decay at large PDI values is associated with the finite size of the basin. That is, the storms with the largest PDI do not have enough room to last a longer time, as their tracks are limited by the size of the basin, which introduces a cutoff in the distribution (at its separation from the power-law fit, roughly) (Supplementary Figs S3-S7). Variations in the definition of the PDI, for example excluding times during which the storm attains subtropical or extratropical status, do not modify the shape of the PDI distribution; the results are also unchanged when restricted to storms that do not make continental landfall (Supplementary Figs S8 and S9).

The degree of similarity between the basins is truly remarkable, given the variety of formative processes at work. TCs in the Western Pacific (typhoons) develop principally from the monsoon, for example, whereas North Atlantic hurricanes are mainly associated with easterly waves (and the degree of association depends on the intensity of the hurricane $)^{8,22}$. In addition, each regional centre or agency follows different protocols in obtaining their data, using techniques that have gradually improved ${ }^{9,11-13}$.

Nevertheless, the shape of the PDI distribution is robust over long time periods. Figure $1 \mathrm{~b}$ shows that this consistency holds over a period of at least 100 years in the North Atlantic (where the record is longest; corrections to the calibration of the maximum velocity do not alter this pattern, Supplementary Fig. S10). Thus, even though the database is certainly incomplete before the satellite era, and even more unreliable before aircraft reconnaissance began in 1944, the fraction of missed storms seems to be independent of PDI. This finding may seem counterintuitive until we consider the fact that a long-lasting TC might be recorded as two shorter storms if its track is lost at some point. A power-law distribution is robust against such splitting of the data (Supplementary Information).

The existence of a simple statistical distribution that describes the whole spectrum of TC dissipation in different basins over a long period of time (apart from incompleteness and finite-size effects) reflects a startling degree of unity in the phenomenon: the small tropical depressions are described in the same way as the most severe fully developed storms. Moreover, a robust power-law distribution is the hallmark of scale invariance ${ }^{17}$. There is no typical TC PDI, up to the maximum possible in a given ocean basin. The fact that all scales are equally important regarding the number of events poses a

${ }^{1}$ Centre de Recerca Matemàtica, Edifici Cc, Campus Universitari, E-08193 Bellaterra, Barcelona, Spain, ${ }^{2}$ Grup de Física Estadística, Facultat de Ciències, Universitat Autònoma de Barcelona, E-08193 Bellaterra, Barcelona, Spain. *e-mail: ACorral@crm.cat. 
a

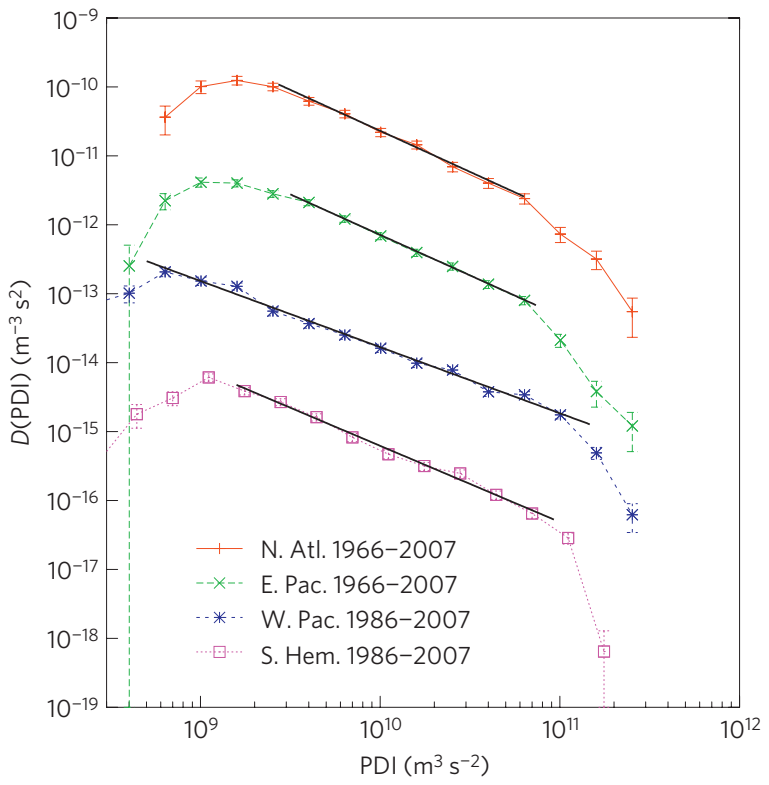

b

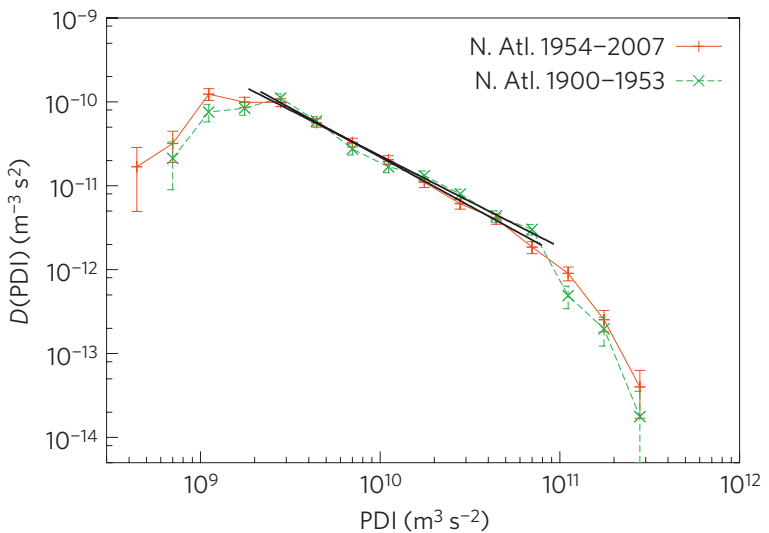

Figure 1 | Power-law distributions of TC PDI values. a, PDI probability densities for TCs in the North Atlantic, Northeastern Pacific, Northwestern Pacific and Southern Hemisphere basins. The period considered is either 1966-2007 or 1986-2007, as indicated in the legend, depending on the reliability of the records and the sufficiency of statistics. The values on the vertical axis are divided by the factors $1, \sqrt{1,000}, 1,000$ and $\sqrt{1,000^{3}}$, to separate the curves for clarity. The distributions are consistent with a power law (straight lines) over some portion of their range, with exponents $\alpha=1.19 \pm 0.06,1.175 \pm 0.05,0.96 \pm 0.02$ and $1.11 \pm 0.04$ from top to bottom, and the Kolmogorov-Smirnov (KS) test yields $p$-values larger than $20 \%$ in all basins (Supplementary Information). Deviations from the power law at large PDI values reflect the finite-size effect. b, PDI probability densities for TCs in the North Atlantic over the 54-year periods 1900-1953 and 1954-2007, with 436 and 579 storms and $\alpha=1.09 \pm 0.04$ and $1.17 \pm 0.04$ (straight lines), respectively. A two-sample KolmogorovSmirnov test (Supplementary Information) gives a $p$-value around $15 \%$ ( $51 \%$ if $\mathrm{PDI}>2 \times 10^{9} \mathrm{~m}^{3} \mathrm{~s}^{-2}$ ). The robustness of the distribution is apparent despite a notable lack of homogeneity in the quality of records.

great challenge to the modelling of this complex phenomenon, and even to large-scale global climate simulations ${ }^{15}$.

Scale invariance can occur in processes where perturbations propagate through a critical (that is, highly susceptible) medium $^{16,18}$. Thus, our result could indicate that the atmosphere, or perhaps the ocean-atmosphere system, is close to a critical state. In fact, this idea is not new; as early as the 1970s it was suggested that atmospheric convection takes place in a near-unstable environment ${ }^{23}$. Much more recently, the existence of a non-equilibrium stability-instability transition to which the state of the atmosphere is attracted has been demonstrated ${ }^{24}$. Some properties of this transition can be obtained from static images of convecting cloud fields ${ }^{25}$ or local observations of precipitation $^{26}$. These findings support our complex-system approach to TC evolution; in correspondence, we provide a complementary perspective to the puzzle that these atmospheric processes constitute. It would be interesting to study whether other nearly universal statistical properties of TCs can be accommodated in this framework ${ }^{27}$.

In addition, an important property of critical systems is that perturbations can evolve while keeping a delicate balance between growth and attenuation, resulting sometimes in sudden intensifications and deintensifications. Although recent years have seen considerable improvement in the prediction of TC trajectories, reliable forecasts of their intensities have not yet been achieved ${ }^{28,29}$. This failure may not be just due to technical limitations; it may be a fundamental feature of the criticality of TC evolution.

In fact, we can draw a strong parallelism between TCs and earthquakes, as the law reported here is analogous to the wellknown Gutenberg-Richter law of seismic occurrence. This law states that, for a given region, the distribution of earthquakes of magnitude $M$ is an exponential function, $D(M) \propto 10^{-b M}$, with $b \simeq 1$; however, in terms of seismic moment, and also in terms of radiated energy $E$, the Gutenberg-Richter law turns out to be a power law, $D(E) \propto 1 / E^{1+2 b / 3}$ (as energy is supposed to be proportional to seismic moment, which is an exponential function of magnitude, that is, $\left.E \propto 10^{3 M / 2}\right)$. Nevertheless, the finite-size effects present in TCs are not so clear in earthquakes ${ }^{30}$.

TC activity shows large interannual variability. One important factor controlling such variability is sea surface temperature (SST). We average SST from the Hadley Center ${ }^{31}$ over the same spatial areas and months in the TC season as in ref. 5 to get an annual (so, seasonal) SST value for each basin; then, we separate the PDI density into two contributions, one for years with SST above its long-term mean value $\langle$ SST $\rangle$ (that is, high SST) and another one for SST below $\langle S S T\rangle$ (low SST). Mathematically, $\langle\mathrm{SST}\rangle \equiv \sum_{y} \mathrm{SST}(y) / Y$, where $\operatorname{SST}(y)$ refers to year $y$ and $Y$ is the total number of years. In this way we eliminate the effect of interannual variations in the number of TCs and concentrate on a comparison of the individual TCs characterizing each type of year.

Remarkably, for the North Atlantic and the Northeastern Pacific, the resulting distributions have essentially the same shape as the distribution for all years but with a difference in scale: high-SST years are characterized by a larger value of the finite-size cutoff, and conversely for low-SST years, as can be seen in Fig. 2a. The other two basins show much minor PDI variation with SST.

If we rescale each conditional distribution by a power of its mean value, $\langle\mathrm{PDI}\rangle$, such that $\mathrm{PDI} \rightarrow \mathrm{PDI} /\langle\mathrm{PDI}\rangle^{v}$ and $D(\mathrm{PDI}) \rightarrow$ $\langle\mathrm{PDI}\rangle^{\beta} D$ (PDI), with $v=\beta=1$ if $\alpha \leq 1$, and $v=1 /(2-\alpha)$ and $\beta=\alpha /(2-\alpha)$ if $\alpha>1$, it becomes apparent that, for each basin, the two distributions share a similar shape, as shown in Fig. $2 b$. (The reason for this rescaling is the fact that a distribution with $\alpha>1$ does not scale linearly with its mean value; see Supplementary Information.) Then, the difference between high-SST and lowSST PDI distributions rests mainly in the scale of the finite-size cutoff and not in the shape of the distribution. A more detailed analysis also shows small changes in the value of the exponents (Supplementary Information).

Years with high SST are thus characterized by hurricanes with larger PDI values. As the PDI integrates the cube of the velocity over the storm lifetime, larger PDI values can result from longer lifetimes, larger (6h) velocities or both. An analysis of the distributions of these variables shows that the increase in PDI comes mainly from an increase in the velocities, most apparent in the range above about 100 knots (that is, corresponding to category 


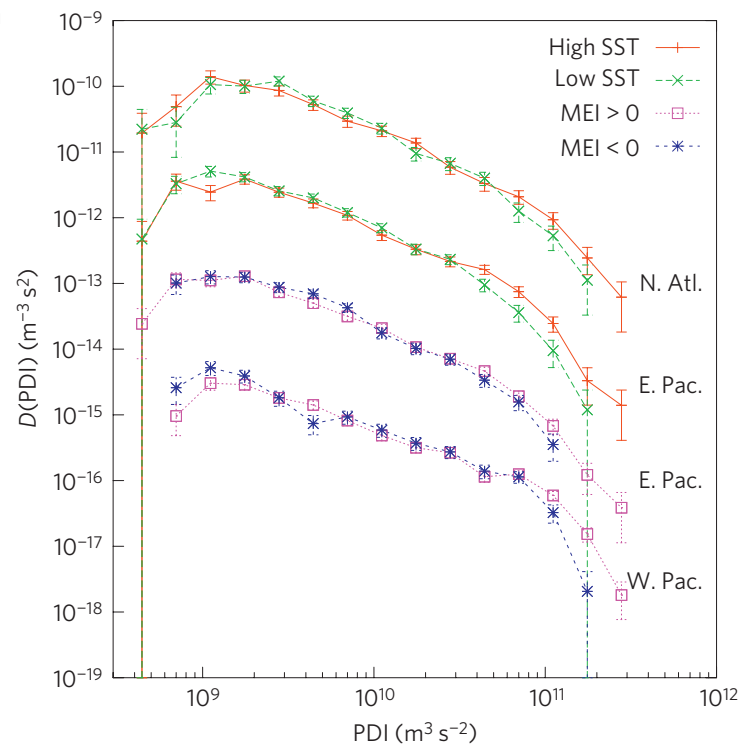

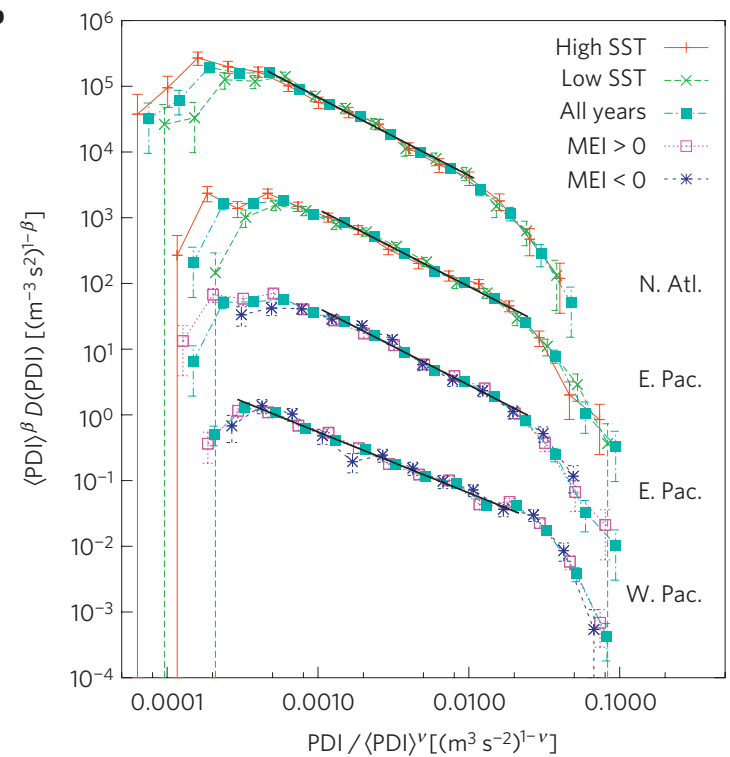

Figure 2 | Scaling of PDI distributions conditioned to SST and EI Niño. a, PDI probability densities calculated separately for years with high or low SST and for years with $\mathrm{MEI}>0$ or $\mathrm{MEI}<0$. Tropical depressions (storms whose maximum $v_{t}$ is below 34 knots, $1 \mathrm{knot}=1.85 \mathrm{~km} \mathrm{~h}^{-1}$ ) are excluded from the Northwestern Pacific dataset, to give all basins the same treatment. Time periods and vertical offsets are as in Fig. 1a. In all cases the data can be fitted by a power law, the worst one being that of the North Atlantic with low SST, which yields $\alpha=1.26 \pm 0.08$ with $p=9 \%$. $\mathbf{b}$, As with the previous panel, except that the unconditioned distributions and their fits are included, and the axes are rescaled by $\langle\text { PDI }\rangle^{\nu(\alpha)}$ and $1 /\langle\text { PDI }\rangle^{\beta(\alpha)}$ with $\alpha=1.19,1.175,1.175$ and $1.0($ the data sets at the bottom have been shifted by extra factors of 100 and $10^{4}$ on each axis for clarity); the ratios of mean PDI between high and low annual values of SST or $\mathrm{MEI},\langle\mathrm{PDI}\rangle_{\text {high }} /\langle\mathrm{PDI}\rangle_{\text {low, }}$ are $1.4,1.6,1.4$ and 1.4, always from top to bottom.

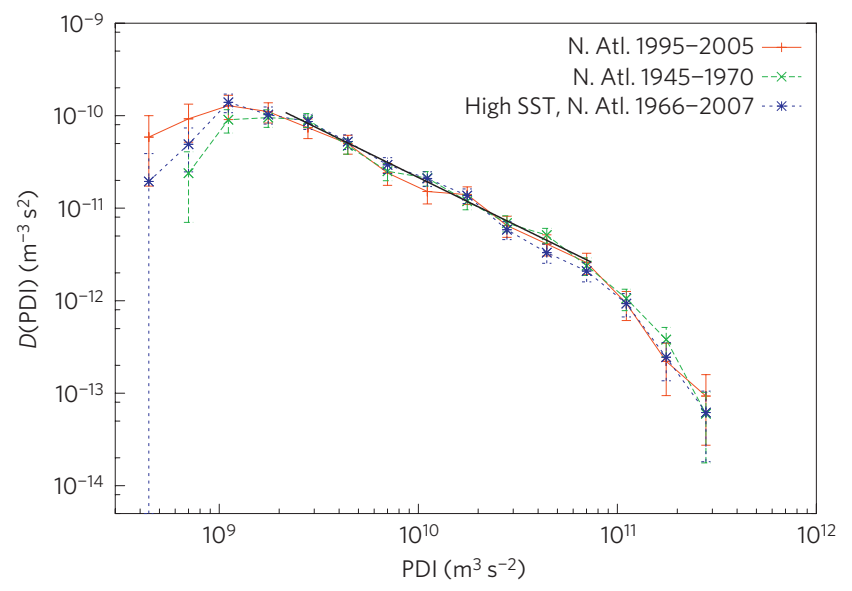

Figure 3 | Equivalence between periods of predominantly high activity and high SST in the North Atlantic. Comparison of PDI probability densities for the years 1995-2005 and 1945-1970, as well as for years with high SST during 1966-2007. The number of storms is 166,257 and 250, and the power-law exponent $\alpha=1.00 \pm 0.06,1.02 \pm 0.07$ and $1.13 \pm 0.08$, respectively. A single fit to the three data sets yields $\alpha=1.05 \pm 0.05$ with $p=92 \%$ (straight line). Two-sample Kolmogorov-Smirnov tests yield $p$-values larger than 35\% for each pair of distributions (Supplementary Table S2), so we can conclude that no significant difference in the energy of TCs can be observed between these periods.

three hurricanes and beyond ${ }^{5}$ ), in comparison with years of low SST (Supplementary Fig. S11).

An analogous study can be done as a function of the socalled MEI index ${ }^{32}$, which quantifies the strength of the El Niño phenomenon. Taking annual values of MEI, years with MEI $>0$ (corresponding to El Niño) lead to increased PDI values in the Northeastern and Northwestern Pacific, but keeping again a similar shape of the PDI distribution, and the opposite when $\mathrm{MEI}<0$, see Fig. $2 a$ and $b$. In the case of the Northwestern Pacific, the contribution of TC lifetimes to the increase in PDI is larger than in the rest of the cases (Supplementary Information). This is somewhat related to the findings of refs 33,34 . Very little variation with MEI is observed in the other two basins. Other indices (AMO, NAO and so on) do not seem to influence the PDI distribution in any basin.

It is a well-known fact that individual years of high (or low) TC activity cluster into longer periods of predominantly high (or low) activity. For example, the North Atlantic has seen extraordinarily high activity between 1995 and 2005, which has been linked to global warming through an increase in the $\mathrm{SST}^{3,5}$ (the issue is nonetheless controversial $\left.{ }^{4,6,11}\right)$. According to our analysis, the PDI distribution for the period 1995-2005 is clearly different from the one for 1971-1994 (in agreement with ref. 3), but it is indistinguishable from the distribution for years with high SST between 1966 and 2007 , as well as from the distribution for other periods of high activity, such as 1945-1970 (ref. 1); see Fig. 3. With the necessary reservations when analysing old hurricane records, we conclude that, so far, the recent dramatic increase of North Atlantic activity has not led to unprecedented energy releases by individual hurricanes.

\section{Methods}

The figures show estimations of the PDI probability density, $D(\mathrm{PDI})$. For any variable quantity $X$, its probability density is defined as $D_{X}(x) \equiv \operatorname{Prob}[x \leq$ variable $<x+\mathrm{d} x] / \mathrm{d} x$, with $\mathrm{d} x \rightarrow 0$ in the ideal case (we omit the label $X$ although for instance for earthquakes $D(M)$ and $D(E)$ are different functions indeed). In practice, $D(x)$ is estimated as the number of cases $n(x)$ between $x$ and $x+\Delta x$ divided by the total number of cases and by $\Delta x$. For power laws and other long-tailed distributions it is recommended not to take a constant $\Delta x$ but a $\Delta x$ that is proportional to $x$ (in our case, $(x+\Delta x) / x \simeq 1.6$ ), which is referred to as logarithmic binning, owing to the fact that in a log-log scale the bins seem to have constant size. Error bars for $D(x)$ can be estimated as $\varepsilon(x) \simeq D(x) / \sqrt{n(x)}$ corresponding to one standard deviation uncertainty (see Supplementary Methods).

Nevertheless, the plots of $D(\mathrm{PDI})$ are only illustrative, as our estimations of the power-law exponent $\alpha$ are independent of the estimation of $D(\mathrm{PDI})$. Instead of fitting a straight line to the log-log plots of $D(\mathrm{PDI})$, we use maximum-likelihood estimation. The goodness of the fit is addressed with the Kolmogorov-Smirnov test (which uses the estimation of the cumulative distribution function) and the 
corresponding $p$-value is obtained from Monte Carlo simulations, as explained in detail in the Supplementary Information.

Received 27 May 2009; accepted 11 June 2010; published online 11 July 2010

\section{References}

1. Goldenberg, S. B., Landsea, C. W., Mestas-Nuñez, A. M. \& Gray, W. M. The recent increase in Atlantic hurricane activity: Causes and implications. Science 293, 474-479 (2001).

2. Trenberth, K. Uncertainty in hurricanes and global warming. Science 308, 1753-1754 (2005).

3. Emanuel, K. Increasing destructiveness of tropical cyclones over the past 30 years. Nature 436, 686-688 (2005).

4. Landsea, C. W. Hurricanes and global warming. Nature 438, E11-E12 (2005)

5. Webster, P. J., Holland, G. J., Curry, J. A. \& Chang, H-R. Changes in tropical cyclone number, duration, and intensity in a warming environment. Science 309, 1844-1846 (2005)

6. Chan, J. C. L. Comment on 'Changes in tropical cyclone number, duration, and intensity in a warming environment'. Science 311, 1713 (2006).

7. Klotzbach, P. J. Trends in global tropical cyclone activity over the past twenty years (1986-2005). Geophys. Res. Lett. 33, L10805 (2006).

8. Shepherd, J. M. \& Knutson, T. The current debate on the linkage between global warming and hurricanes. Geogr. Compass 1, 1-24 (2007).

9. Kossin, J. P., Knapp, K. R., Vimont, D. J., Murnane, R. J. \& Harper, B. A. A globally consistent reanalysis of hurricane variability and trends. Geophys. Res. Lett. 34, L04815 (2007).

10. Elsner, J. B., Kossin, J. P. \& Jagger, T. H. The increasing intensity of the strongest tropical cyclones. Nature 455, 92-95 (2008).

11. Gray, W. M. Comments on 'Increasing destructiveness of tropical cyclones over the past 30 years'. Preprint at http://arxiv.org/abs/physics/0601050 (2006)

12. Landsea, C. W., Harper, B. A., Hoarau, K. \& Knaff, J. A. Can we detect trends in extreme tropical cyclones? Science 313, 452-454 (2006).

13. Landsea, C. W. Counting Atlantic tropical cyclones back to 1900. Eos 88, 197-202 (2007).

14. Emanuel, K. Divine Wind: The History and Science of Hurricanes (Oxford Univ. Press, 2005).

15. Emanuel, K. The hurricane-climate connection. Bull. Am. Meteorol. Soc. 5, ES10-ES20 (2008).

16. Bak, P. How Nature Works: The Science of Self-Organized Criticality (Copernicus, 1996).

17. Christensen, K. \& Moloney, N. R. Complexity and Criticality (Imperial College Press, 2005).

18. Malamud, B. D. Tails of natural hazards. Phys. World 17, 31-35 (2004).

19. Kantha, L. Time to replace the Saffir-Simpson hurricane scale? Eos 87, 3-6 (2006).

20. Jarvinen, B. R., Neumann, C. J. \& David, M. A. S. A tropical cyclone data tape for the North Atlantic basin, 1886-1983: Contents, limitations, and uses. http://www.nhc.noaa.gov/pdf/NWS-NHC-1988-22.pdf (1988).

21. Chu, J-H., Sampson, C. R., Levine, A. S. \& Fukada, E. The joint typhoon warning center tropical cyclone best-tracks, 1945-2000. https://metocph.nmci.navy.mil/jtwc/best_tracks/TC_bt_report.html (2002).
22. Landsea, C. W. A climatology of intense (or major) hurricanes. Mon. Weath. Rev. 121, 1703-1713 (1993).

23. Arakawa, A. \& Schubert, W. H. Interaction of a cumulus cloud ensemble with the large-scale environment, part I. J. Atmos. Sci. 31, 674-701 (1974).

24. Peters, O. \& Neelin, J. D. Critical phenomena in atmospheric precipitation. Nature Phys. 2, 393-396 (2006).

25. Peters, O., Neelin, J. D. \& Nesbitt, S. W. Mesoscale convective systems and critical clusters. J. Atmos. Sci. 2913-2924 (2009).

26. Peters, O., Hertlein, C. \& Christensen, K. A complexity view of rainfall. Phys. Rev. Lett. 88, 018701 (2002).

27. Emanuel, K. A statistical analysis of tropical cyclone intensity. Mon. Weath. Rev. 128, 1139-1152 (2000).

28. Willoughby, H. E. Forecasting hurricane intensity and impacts. Science 315, 1232-1233 (2007).

29. National Hurricane Center Forecast Verification. http://www.nhc.noaa.gov/verification (2008).

30. Main, I. G., Li, L., McCloskey, J. \& Naylor, M. Effect of the Sumatran mega-earthquake on the global magnitude cut-off and event rate. Nature Geosci. 1, 142 (2008).

31. Rayner, N. A. et al. Global analyses of sea surface temperature, sea ice, and night marine air temperature since the late nineteenth century. J. Geophys. Res. 108, 4407 (2003).

32. Wolter, K. \& Timlin, M. S. Measuring the strength of ENSO events: How does 1997/98 rank? Weather 53, 315-324 (1998).

33. Lander, M. A. An exploratory analysis of the relationship between tropical storm formation in the Western North Pacific and ENSO. Mon. Weath. Rev. 122, 636-651 (1994).

34. Emanuel, K. Environmental factors affecting tropical cyclone power dissipation. J. Clim. 20, 5497-5509 (2007)

\section{Acknowledgements}

We have benefited from the expertise and kindness of A. Clauset, A. Deluca, K. Emanuel, E. Fukada, A. González, J. Kossin, R. D. Malmgren, B. Mathiesen, M. Paczuski, O. Peters, G. B. Raga, R. Romero and A. Turiel. A.O. and A.C. were put in contact through G. Orriols. The initial part of our research was financed by the Explora-Ingenio 2010 program, grant FIS2007-29088-E, and also partially by FIS2009-09508, CGL2007-60797/CLI and 2009SGR-164. A.C. is also a participant of the Consolider i-Math project.

\section{Author contributions}

A.O. downloaded the data, A.C. wrote most of the codes, A.O. and A.C. analysed the data, A.C. wrote the paper, J.E.L. provided feedback and support and participated in discussions.

\section{Additional information}

The authors declare no competing financial interests. Supplementary information accompanies this paper on www.nature.com/naturephysics. Reprints and permissions information is available online at http://npg.nature.com/reprintsandpermissions. Correspondence and requests for materials should be addressed to A.C. 